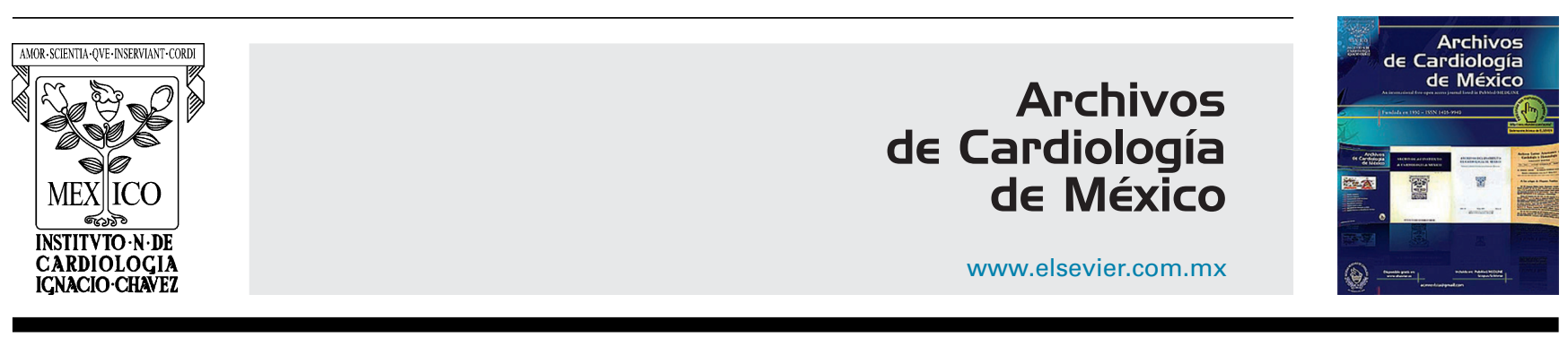

CARTAS CIENTÍFICAS

\section{Utilidad del ECMO veno-arterial en el manejo posquirúrgico del choque cardiogénico refractario en enfermedad aórtica compleja}

\section{Usefulness of venous-arterial extracorporeal membrane oxygenation in post-surgical management of refractory cardiogenic shock in complex aortic disease}

\section{Introducción}

La mortalidad de la disección aórtica en síndrome de Marfan (SM) es del $90 \%$ por lo que el manejo quirúrgico urgente con cirugía de reemplazo de raíz y aorta ascendente con injerto tubular valvulado denominado procedimiento de Bentall y de Bono (PBB) se considera de elección en estos pacientes. Esta técnica ha demostrado baja morbimortalidad con una sobrevida a los 5 y 10 años del $70-80 \%$ y del $50-70 \%$, respectivamente; sin embargo, la mortalidad temprana está asociada a la presencia de choque cardiogénico el cual incrementa la mortalidad hasta 4 veces con un porcentaje de hasta el $80 \%^{1,2}$.

McCarthy et al. en un metaanálisis del uso de asistencia ventricular mediante oxigenación por membrana extracorpórea (ECMO) en choque cardiogénico, reportó que el choque cardiogénico poscardiotomía tiene la mortalidad más alta de todas las indicaciones del uso de la ECMO y en un análisis a 15 años del registro Extracorporeal Life Support Organization (ELSO) se reportó una sobrevida de tan solo del $15 \%^{3,4}$.

A pesar de los desalentadores resultados en la sobrevida de los pacientes con choque poscardiotomía con asistencia ventricular, este manejo está considerado en las guías actuales de tratamiento de la insuficiencia cardiaca aguda con una clase IIb, nivel de evidencia $C^{5}$.

\section{Presentación del caso}

Mujer de 38 años con diagnóstico definitivo de SM. Ingresó al servicio de urgencias por dolor torácico típico de disección aórtica. Se le realizó una angiotomografía la cual reportó dilatación de aorta ascendente de $56.6 \mathrm{~mm}$ y disección aórtica desde la raíz a nivel de los senos de Valsalva con flap de disección extendido al tronco braquiocefálico, aorta ascendente, arco aórtico, aorta descendente inmediatamente posterior al istmo, disección del segmento proximal de la carótida común derecha. Se le realizó PBB con prótesis aórtica biológica tipo INC, sustitución del arco aórtico, revascularización de troncos supraaórticos (revascularización extra anatómica de la subclavia izquierda) y reimplante de arterias coronarias. Se decidió utilizar un injerto valvulado fabricado en nuestro instituto con una válvula de manufactura propia de pericardio bovino además de un tubo de Dacron para la aorta ascendente y arco aórtico y no un injerto prefabricado porque en nuestra experiencia institucional, esto reduce los costes económicos y ofrece la misma utilidad. Tuvo un tiempo de circulación extracorpórea de 272 min y pinzamiento aórtico de $197 \mathrm{~min}$. Evolucionó con hemorragia mayor a lo habitual, vasoplejía y choque cardiogénico. Se le realizó un ecocardiograma transtorácico en el cual se observó alteraciones del engrosamiento global y FE del 15\% (fig. 1). Su perfil hemodinámico inicial con IC 1.3, PCP 15 y posteriormente, a pesar de optimizar precarga y del manejo con noradrenalina $40 \mu \mathrm{g} / \mathrm{min}$, vasopresina $10 \mathrm{Ul} / \mathrm{h}$, dobutamina $10 \mu \mathrm{g} / \mathrm{kg} / \mathrm{min}$ y levosimendán $0.2 \mu \mathrm{g} / \mathrm{kg} / \mathrm{min}$ presentó hipotensión y bajo gasto cardiaco persistente, motivo por el cual se decidió colocar balón intraaórtico de contrapulsación y ECMO veno-arterial con canulación periférica femoral la cual fue realizada en quirófano. No se colocó cánula de reperfusión y no hubo complicaciones isquémicas del miembro pélvico documentadas; la decisión de realizar un abordaje femoral fue considerado por el equipo quirúrgico dado que la disección no se extendía a la aorta abdominal. La utilidad de utilizar ambos dispositivos fue disminuir la poscarga impuesta por la canulación de la ECMO. Los parámetros del oxigenador de la ECMO se ajustaron para obtener valores de saturación venosa mixta de $\mathrm{O}_{2}>70 \%, \mathrm{PaCO}_{2}: 37-42 \mathrm{mmHg}, \mathrm{PaO}_{2}$ en la sangre a la salida del oxigenador $>300 \mathrm{mmHg}$. Se anticoaguló con heparina no fraccionada en infusión continua con ajustes precisos para mantener un tiempo de coagulación activado (TCA) entre 160-180 s. Esta anticoagulación se inició después de las primeras $24 \mathrm{~h}$ para evitar hemorragia y el soporte ventricular se mantuvo durante $72 \mathrm{~h}$ en conjunto con manejo presor, inotrópico y balón de contrapulsación intraaórtico de 40 cc el cual se mantuvo por $72 \mathrm{~h}$ más una vez retirado la ECMO. Evolucionó con lesión renal aguda con requerimiento de terapia lenta continua y posteriormente hemodiálisis intermitente. Presentó hígado de choque, coagulopatía por consumo, falla respiratoria, neumonía nosocomial, choque séptico, miopatía del enfermo en estado crítico. Tras el fracaso multiorgánico y la resolución paulatina del mismo, tuvo 


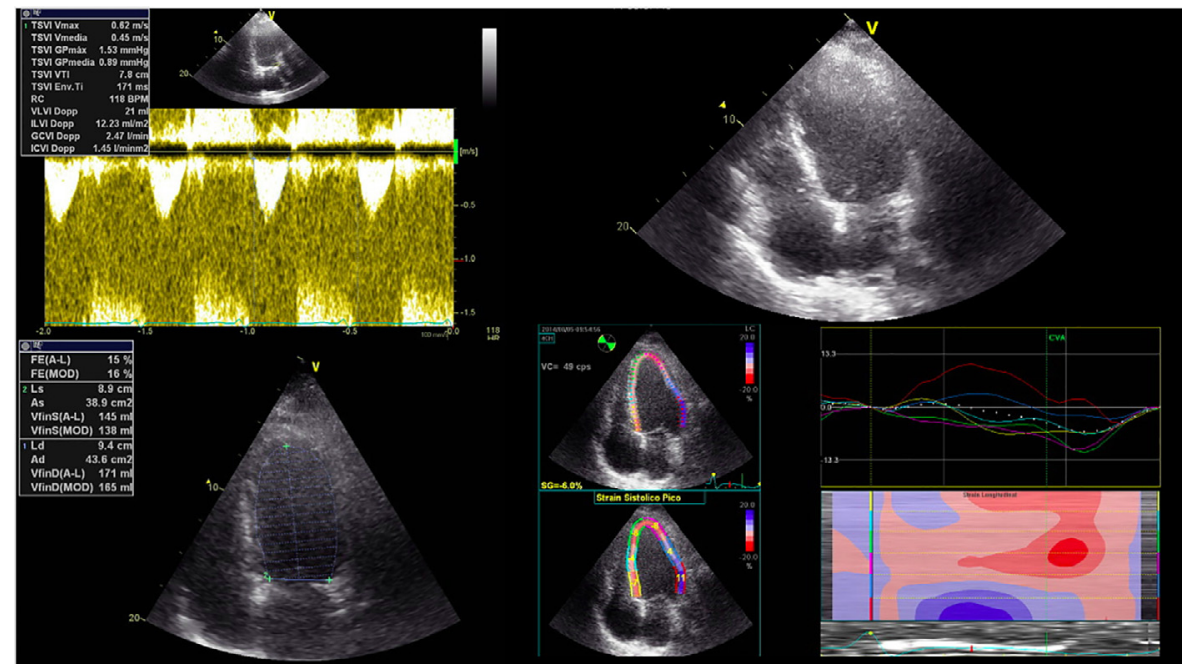

Figura 1 Ecocardiograma transtorácico pre-ECMO. Se puede observar una dilatación importante del ventrículo izquierdo con una FEVI del $15 \%$, Strain global $-6 \%$, ITV del tracto de salida del ventrículo izquierdo $7.8 \mathrm{~cm}$.

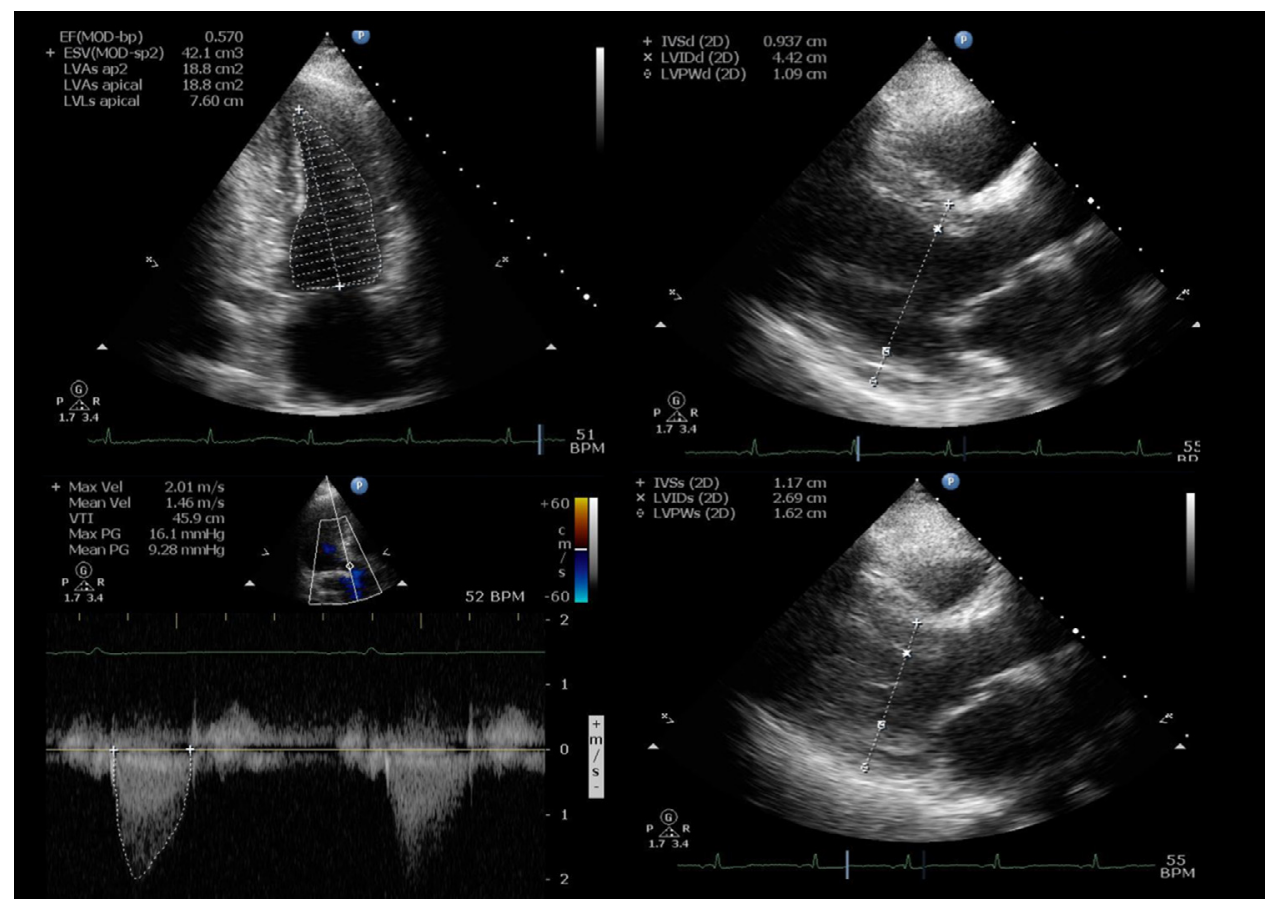

Figura 2 Ecocardiograma 2016. Se observa una FEVI del 57\%, diámetros conservados, movilidad, engrosamiento normal en reposo, prótesis aórtica normofuncionante.

una hospitalización prolongada de 4 meses. Fue egresada a su domicilio con una FEVI del $10 \%$ con tratamiento médico para falla cardiaca y rehabilitación.

Actualmente, la paciente se encuentra en fase III de rehabilitación cardiaca en clase funcional I. Su ecocardiograma de 2016, reportó una FEVI del 56\%, cavidades cardiacas sin dilatación, movilidad global y segmentaria en reposo normal y prótesis biológica aórtica normofuncionante (fig. 2).

\section{Discusión}

El choque cardiogénico poscardiotomía complica el 2-6\% de todos los casos de cirugía cardiaca y es caracterizado por el desarrollo de un síndrome de bajo gasto cardiaco e hipoperfusión en el periodo postoperatorio temprano. Se define como refractario cuando cursa con hipoperfusión tisular, presión arterial sistólica menor a $90 \mathrm{mmHg}$ e índice cardiaco menor a $2 \mathrm{l} / \mathrm{min} / \mathrm{m}^{2}$ a pesar del manejo con balón de contrapulsación intraaórtico, soporte inotrópico y vasopresor, con una mortalidad hospitalaria del $50-80 \%$ y el manejo se orienta a la implantación de dispositivos de asistencia ventricular como en este caso. Los factores de riesgo para presentarlo son la edad avanzada, disfunción ventricular izquierda preexistente y tiempos prolongados de circulación extracorpórea y pinzamiento aórtico. El choque cardiogénico prequirúrgico es un factor independiente de mortalidad 
con RR 2.6 ${ }^{6}$. El tratamiento con soporte ventricular ECMO veno-arterial temprano es crucial en el tratamiento; provee un soporte hemodinámico biventricular completo con la ventaja principal de su implementación periférica rápida en la cama del paciente ${ }^{7,8}$.

El caso presentado muestra un paciente con SM y enfermedad aórtica compleja manejada con PBB y reconstrucción de arco aórtico con evolución con choque cardiogénico refractario el cual fue manejado según guías mexicanas e internacionales de tratamiento de la falla cardiaca aguda, inicialmente con optimización del entorno metabólico y de las determinantes del gasto cardiaco, tratamiento guiado por objetivos y monitorización hemodinámico invasivo con catéter Swan-Ganz, así como monitorización hemodinámica no invasiva guiada por ecocardiografía transtorácica. La ausencia de mejoría hemodinámica tras $30 \mathrm{~min}$ de apoyo presor e inotrópico nos obligó a colocar balón de contrapulsación intraaórtico y la ausencia de mejoría en las siguientes horas, a implementar asistencia ventricular con ECMO veno-arterial ${ }^{9}$. La evolución hemodinámica fue buena, sin embargo, no suficiente para evitar la falla multiorgánica y las complicaciones crónicas e infecciosas esperadas de una hospitalización prolongada; sin embargo, el manejo multidisciplinario y la rehabilitación cardiaca iniciada culminaron con la recuperación total de la enferma.

Enfatizamos la importancia del manejo guiado por objetivos con implante temprano de dispositivos de asistencia ventricular para mejorar la atención y el pronóstico de estos pacientes.

\section{Bibliografía}

1. Podolec P, Tracz W, Kostkiewicz M, et al. Clinical and echocardiographical study of the aortic homograft implantations in patients with Marfan syndrome. Int J Cardiol. 1997;60:41-7.

2. Miler W Jr, Warren WD, Blanton FS. A method for resection of the aortic arch. Ann Surg. 1960;151:225-30.

3. McCarthy FH, McDermott KM, Kini V, et al. Trends in U.S. extracorporeal membrane oxygenation use and outcomes: 2002-2012. Semin Thorac Cardiovasc Surg. 2015;27:81-8.
4. Whitman GJ. Extracorporeal membrane oxygenation for the treatment of postcardiotomy shock. J Thorac Cardiovasc Surg. 2017;153:95-101.

5. Ponikowski P, Voors A, Anker S, et al. 2016 ESC Guidelines for the diagnosis and treatment of acute and chronic heart failure: The Task Force for the diagnosis and treatment of acute and chronic heart failure of the European Society of Cardiology (ESC)Developed with the special contribution of the Heart Failure Association (HFA) of the ESC. Eur Heart J. 2016;37:2129-200.

6. Martín CE, Forteza A, Pérez E, et al. Factores predictores de mortalidad y reoperación en la cirugía de la disección aórtica aguda tipo A: 18 años de experiencia. Rev Esp Cardiol. 2008;61:1050-60.

7. Thiele H, Zeymer U, Neumann FJ, et al. Intraaortic balloon support for myocardial infarction with cardiogenic shock. N Engl J Med. 2012;367:1287-96.

8. Reyentovich A, Barghash MH, Hochman JS. Management of refractory cardiogenic shock. Nat Rev Cardiol. 2016:481-92.

9. Reyes M, Carrillo J, Hernández M, et al. Guía de la práctica clínica basada en la evidencia para el manejo posquirúrgico del paciente llevado a cirugía cardiaca. Arch Cardiol Mex. 2011;81 Supl 2:S30-40.

Alejandra Rodríguez-Hernández ${ }^{a, *}$, Carlos Adán-Brito ${ }^{b}$, Gustavo Rojas-Velasco ${ }^{a}$ y Francisco Martín Baranda-Tovar ${ }^{a}$

a Terapia Intensiva Cardiovascular, Instituto Nacional de Cardiología Ignacio Chávez, Ciudad de México, México

${ }^{\mathrm{b}}$ Ecocardiografía, Instituto Nacional de Cardiología Ignacio Chávez, Ciudad de México, México

* Autor para correspondencia. Juan Badiano 1, Col. Belisario Domínguez Sección XVI, Delegación Tlalpan, 14080, Ciudad de México. Teléfono: 55-73-29-11, Ext 1504.

Correo electrónico: alejandrarh1985@hotmail.com

(A. Rodríguez-Hernández).

https://doi.org/10.1016/j.acmx.2017.07.001

1405-9940/

(c) 2017 Instituto Nacional de Cardiología Ignacio Chávez. Publicado por Masson Doyma México S.A. Este es un artículo Open Access bajo la licencia CC BY-NC-ND (http: / / creativecommons.org/ licenses/by-nc-nd/4.0/).

\section{Masa auricular derecha en paciente con mieloma múltiple}

\section{Right auricular mass in a patient with multiple myeloma}

\section{Introducción}

Las neoplasias originadas de las células plasmáticas pueden presentarse como lesiones solitarias (plasmocitoma) o múltiples (mieloma múltiple). En los casos de plasmocitoma solitario se desarrollan de forma predominante en la médula ósea o, menos habitualmente, en tejidos blandos (plasmocitoma extramedular), siendo con mayor frecuencia en cabeza y cuello.

Para el diagnóstico de plasmocitoma extramedular es preciso demostrar, junto a la presencia de un clon de células plasmáticas en la biopsia de la tumoración, la ausencia de lesiones líticas, aspirado medular sin plasmocitosis y ausencia de hipercalcemia, insuficiencia renal y anemia.

Es muy poco habitual la afectación cardiaca por este tipo de neoplasias durante su historia natural. Mostramos un extraño caso de infiltración cardiaca por este grupo de tumores.

\section{Descripción del caso}

Varón de 82 años, con antecedente de hipertensión arterial bien controlada con 2 fármacos e hipertrofia benigna de próstata. Comenzó con una tumoración pectoral derecha de rápido crecimiento, cuyo análisis anatomopatológico correspondió a plasmocitoma extramedular. Los estudios complementarios practicados mostraron una función renal normal, calcio total de $8.2 \mathrm{mg} / \mathrm{dl}$, proteínas totales $7.1 \mathrm{~g} / \mathrm{dl}$, 